\title{
The prognostic value of Krebs von den Lungen- 6 and surfactant protein-A levels in the patients with interstitial lung disease
}

\section{Peiyan Zheng ${ }^{1 *}$, Xiaomao Zheng ${ }^{1 *}$, Hasegawa Takehiro ${ }^{2}$, Zhangkai Jason Cheng ${ }^{1}$, Jingxian Wang ${ }^{1,3}$, Mingshan Xue ${ }^{1}$, Quanming Lin $^{1}$, Zhifeng Huang ${ }^{1}$, Huimin Huang ${ }^{1}$, Chenxi Liao ${ }^{1}$, Baoqing Sun ${ }^{1}$ \\ 'Department of Allergy and Clinical Immunology, Guangzhou Institute of Respiratory Health, State Key Laboratory of Respiratory Disease, National Clinical Research Center of Respiratory Disease, The First Affiliated Hospital of Guangzhou Medical University, Guangzhou 510120, Guangdong Province, China; \\ ${ }^{2}$ Sysmex R\&D Center Europe GmbH, Falkenried 88, 20251 Hamburg, Germany; \\ ${ }^{3}$ National Joint Local Engineering Laboratory for Cell Engineering and Biomedicine Technique, Guizhou Province Key Laboratory of Regenerative Medicine, Key Laboratory of Adult Stem Cell Translational Research (Chinese \\ Academy of Medical Sciences), Guizhou Medical University, Guiyang 550025, Guizhou Province, China.}

\section{ABSTRACT}

Background and Objectives: The highly variable clinical course of interstitial lung disease (ILD) makes it difficult to predict patient prognosis. Serum surfactant protein-A (SP-A) and Krebs von den Lungen-6 (KL-6) are known prognostic biomarkers. However, the clinical or pathophysiological differences in patients with these biomarkers have not been well evaluated. We investigated the clinical and pathophysiological differences through the comparison of SP-A and KL-6 levels before and after treatment. Methods: This study included retrospective data from 91 patients who were treated for ILD between August 2015 and September 2019. Serum SP-A and KL-6 levels were measured before and after treatment. The patients were followed up for 3 months. Results: Changes in the serum biomarkers (Delta SP-A and Delta KL-6) were found to be significantly correlated ( $r s=0.523, P<0.001$ ); Delta SP-A and Delta KL-6 were inversely correlated with changes in pulmonary function (\% predicted values of diffusing capacity for carbon monoxide [DLCO], forced vital capacity [FVC], and forced expiratory volume in $1 \mathrm{~s}$ [FEV1]). Patients were divided into four groups based on their Delta SP-A and Delta KL-6 levels in a cluster analysis (G1, G2, G3, and G4). Both SP-A and KL-6 were elevated in the G1 group, with all the patients enrolled classified as progressive or unchanged, and $86.4 \%$ of patients showed improved disease activity in the G4 group, where both SP-A and KL-6 levels were reduced. In the G2 group, only SP-A levels decreased post-treatment, indicating an improvement in respiratory function; the patients were not at the end stage of the disease. Only the SP-A levels increased in the G3 group with immunosuppressive treatment. Conclusions: Reduced serum SP-A and/or KL-6 levels are associated with improved lung function in patients with ILD. Some patients only showed a decrease in SP-A levels could prognosis an improvement in respiratory function. When only SP-A is increased, it may imply that the patients are at an early stage of disease progression. As a result, for proper disease monitoring, measuring both markers is important.

Key words: interstitial lung disease, Krebs von den Lungen-6, surfactant protein-A, diffusing capacity for carbon monoxide, forced vital capacity

\section{INTRODUCTION}

Interstitial lung disease (ILD) comprises a group of acute and chronic lung diseases characterized by diffuse pulmonary parenchyma, alveolar inflammation, and interstitial fibrosis. ILD includes both common and rare clinical diseases. In most cases, the causes remain unclear. ILD can be life-threatening, with an overall mortality 
rate as high as $52 \%{ }^{[1]}$ Appropriate diagnosis and prognosis are essential for patients with ILD.

The present ILD diagnosis depends on pulmonary function tests (PFTs), radiological and histological examinations, and high-resolution computed tomography (HRCT). ${ }^{[2]}$ These methods provide important information for the treatment of ILD. However, the pathophysiology of ILD is complex. Various biomarkers are used to support both the diagnosis of ILD and its prognosis evaluation, as they are potentially helpful in identifying vulnerable patients. Among these markers are the noninvasive serum biomarkers surfactant protein-A (SP-A) and Krebs von den Lungen-6 (KL-6), which have been found to provide particularly important reference values for diagnosing ILD, monitoring disease activity, and evaluating prognosis. ${ }^{[3-5]}$

SP-A and KL-6 are produced in the alveoli and released into the bloodstream when the alveoli are destroyed, causing their levels to increase as the disease worsens and decrease as the patient recovers. ${ }^{[4,6-8]}$ It has been reported that serum levels of SP-A and KL-6 vary with different disease types and show different correlations with the ground-glass opacity grade and honeycomb lung grade derived from HRCT imaging. ${ }^{[4,9-14]}$ SP-A and KL-6 are also used as blood biomarkers to predict the prognosis of idiopathic with pulmonary fibrosis (IPF) patients, ${ }^{[15,16]}$ and SP-A has been identified as a biomarker for various lung diseases, including acute respiratory distress syndrome, chronic obstructive pulmonary disease, and progressive systemic sclerosis. ${ }^{[17]}$ Measuring and monitoring serum KL-6 concentrations have been reported to be useful for screening ILD and determining its severity. ${ }^{[18-21]}$ In Japan, serum SP-A and KL-6 levels are widely used as biomarkers for ILD diagnosis, severity assessment, and prediction of clinical outcomes. A cut-off value for serum KL-6 of 500 $\mathrm{U} / \mathrm{mL}$ has been used in clinical practice to distinguish patients with ILD from healthy individuals or non-ILD patients with pulmonary disease. ${ }^{[3,4]}$

Despite the increasing number of publications on SP-A and KL-6 in the context of ILD, there have been no detailed studies on the differences between the two biomarkers. This study investigated the characteristics of serum SP-A and KL-6 levels in patients with ILD, comparing the levels before and after treatment, to explore the monitoring value of these biomarkers for predicting ILD prognosis in Chinese patients.

\section{METHODS}

\section{Subjects}

This retrospective study included data from 91 patients with ILD who were treated at the First Affiliated Hospital of Guangzhou Medical University between August 2015 and September 2019, and included 71 healthy volunteers as the control group. This study was approved by the Ethical Committee of the First Affiliated Hospital of Guangzhou Medical University (ethics approval no. gyfyy-2016-73). All procedures were performed in accordance with the guidelines and regulations of the ethics committee. Written informed consent was obtained from all patients.

All patients with ILD were diagnosed using HRCT, PFT, serologic domain with specific autoantibodies, and clinical systems. Idiopathic interstitial pneumonia (IIP) was classified according to the 2013 American Thoracic Society/European Respiratory Society (ATS/ERS) consensus, ${ }^{[22]}$ and interstitial pneumonia with autoimmune features was diagnosed according to the 2015 ATS/ERS consensus. ${ }^{[2]}$ The diagnostic criteria for connective tissue disease associated with ILD (CTD-ILD), IPF, hypersensitivity pneumonitis (HP), and other types of ILD were based on the clinical and/or serological domain criteria specified by the ERS/ATS task force. The patients in this study included those with CTD-ILD, IIP, interstitial pneumonia with autoimmune features (IPAF), HP, and unclassifiable ILD. CTD-ILD included rheumatoid arthritis, systemic sclerosis, primary Sjogren's syndrome, polymyositis, dermatomyositis, systemic lupus erythematosus, and mixed connective tissue disease. IIP included IPF, nonspecific interstitial pneumonia, desquamative interstitial pneumonia, cryptogenic organizing pneumonia, and acute interstitial pneumonia. The patients were either treated with immunosuppressants or with corticosteroids according to the internationally accepted guidelines and their clinical symptoms (i.e., glucocorticoids alone or in combination with azathioprine, cyclophosphamide, pirfenidone, etc.). Patients with other connective tissue diseases, autoimmune diseases, malignancies, infectious diseases, or drug-induced ILD; pregnant and lactating women; and patients $<18$ years were excluded.

\section{Collection of clinical information and serum samples}

Blood samples were collected before and after treatment to measure the serum levels of SP-A and KL-6. The serum was separated and stored at $-80{ }^{\circ} \mathrm{C}$ until analysis. All patients were followed up for at least 3 months. The clinical follow-up data were collected from the patients' medical records: sex, age, body mass index (BMI), smoking history, white blood cell (WBC) count, neutrophil (NEUTP), monocyte (MONO), eosinophil (EOP), and basophil (BASOP) ratios; C-reactive protein (CRP) and lactate dehydrogenase (LDH) levels; therapeutic drugs used; and pulmonary function.

\section{Pulmonary function tests}

Where possible, lung function parameters, including forced vital capacity (FVC), forced expiratory volume 
in $1 \mathrm{~s}$ (FEV1), and diffusing capacity for carbon monoxide (DLCO), were measured in accordance with the Standardization of Spirometry guidelines. However, some patients with severe ILD were unable to undergo the PFT.

\section{Measurement of serum SP-A and KL-6 levels}

Serum levels of SP-A and KL-6 were measured using a fully automatic immunoanalyzer, the HISCL-5000 (Sysmex Corp., Hyogo, Japan), according to the manufacturer's instructions. The detection range for SP-A was 1-1000 ng/ $\mathrm{mL}$ and for KL-6 was 10-6000 U/mL. Results higher than the upper detection limit were excluded from the analysis. The cut-off concentrations were $43.8 \mathrm{ng} / \mathrm{mL}$ and $500 \mathrm{U} /$ $\mathrm{mL}$, respectively. SP-A and KL-6 assay kits were provided by Sysmex Corporation (Kobe, Japan).

\section{Definitions of disease progression, improvement, and no change}

Disease progression was defined as a decline in FVC by $\geq 10 \%$ and/or a decrease in DLCO by $\geq 15 \%$. Disease improvement was defined as an increase in FVC by $\geq 10 \%$ and/or an increase in DLCO by $\geq 15 \%$. No change in condition was defined as an FVC change of $<10 \%$ and a DLCO change of $<15 \%$.

\section{Statistical analyses}

The normality of the continuous variable distributions was tested using the Shapiro-Wilk test, and the data were expressed as mean \pm standard deviation for normal distributions or as median with interquartile range for non-normal distributions. Dichotomous variables are presented as frequencies and percentages. The chi-squared test was used to analyze the differences in categorical data. Differences in the serum marker levels between the patient groups were analyzed using the Wilcoxon signed-rank test and Fisher's exact test. Other differences between the three patient groups were analyzed using the Kruskal-Wallis test, Steel-Dwass test, or Fisher's exact test. Correlation analyses were performed using Spearman's rank correlation analysis. The calculated values were standardized, and an unsupervised hierarchical cluster analysis was performed using Cluster 3.0 (University of Tokyo Human Genome Center). Statistical analyses were performed using SPSS for Windows (version 22.0; IBM Corp., Armonk, NY, USA) and Cluster 3.0 (University of Tokyo Human Genome Center). Statistical significance was set at $P<0.05$.

\section{RESULTS}

\section{Basic information about the study subjects}

The results revealed no significant differences in age and gender between patients with ILD and healthy controls. Serum SP-A and KL-6 levels were significantly higher in ILD patients than in healthy controls $(P<0.001)$ (Table S1).
Of the 91 patients with ILD included in this study, 36 $(39.56 \%)$ were diagnosed with CTD-ILD, 20 (21.98\%) with IIP, $22(24.18 \%)$ with IPAF, $4(4.39 \%)$ with HP, and $9(9.89 \%)$ with unclassified ILD. Table S2 shows no significant differences in serum SP-A and KL-6 levels in different pathological types of ILDs pretreatment and post-treatment, that is, among patients with CTD-ILD, IIP, IPAF, and others (patients with HP or with unclassified ILD). Figure S1 shows a flowchart of allocation of the patients to progressive, improved, and unchanged groups, according to the change in pulmonary function before and after treatment. After treatment, $27(29.67 \%)$ of the cases were classified as progressive, $36(39.56 \%)$ as improved, and $28(30.77 \%)$ as unchanged groups.

Baseline clinical characteristics of the patients are shown in Table 1. The patients ranged in age from 19 to 80 years, with a median age of 55 (45-64) years; $42(46.2 \%)$ were male and $22(24.18 \%)$ were smokers. The median followup time was 8.2 months. The median values for the lung function parameters ( $\%$ predicted values for FVC, FEV1, and DLCO) were lower than the normal range. There were no significant differences in any of the parameters among the three groups.

\section{Clinical and laboratory parameters pretreatment and post-treatment}

Figure 1A-I summarizes the pretreatment and posttreatment values of CRP, LDH, WBC count, NEUTP ratio, MONO ratio, EOP ratio, and the PFT parameters in the three groups. There was no statistically significant difference in CRP, LDH, WBC count, and NEUTP ratio pretreatment and post-treatment in the three groups (Figure 1A-D). MONO and EOP ratios showed a significant decrease in the improved group $(P=0.024$ and $P=0.005$, respectively; Figure $1 \mathrm{E}$ and $\mathrm{F}$, respectively). The three pulmonary function parameters $(\%$ predicted values of DLCO, FVC, and FEV1) significantly improved in the improved group; all these parameters were significantly reduced in the progressive group, and these deteriorations and improvements were not statistically significant in the unchanged group $(P>0.05)$ (Figure 1G-I).

\section{Serum SP-A and KL-6 levels before and after treatment}

Pretreatment serum levels of SP-A and KL-6 were not significantly different among the progressive, improved, and unchanged groups (Table 1).

Comparison of SP-A and KL-6 levels before and after treatment in the progressive group showed that there was a significant increase in median serum SP-A levels from 41.7 (33.5-55.9) ng/mL to 75.1 (37.6-90.6) ng/mL $(P<0.05)$ and in median serum KL-6 levels from 1070 (738-2117) 
A

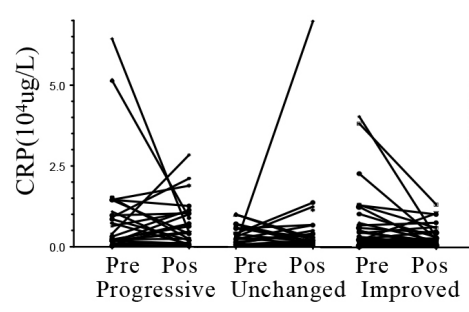

D

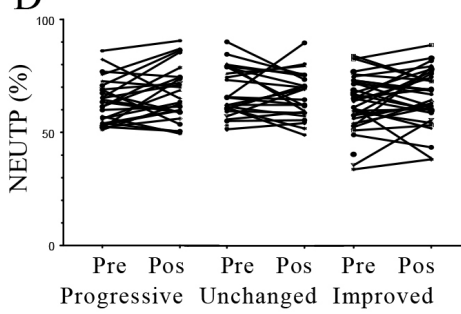

B

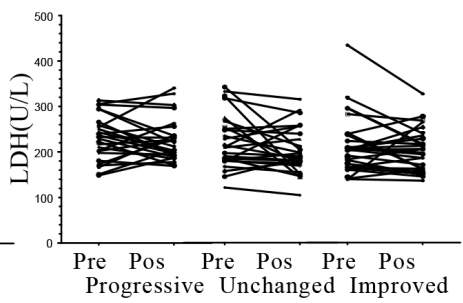

E

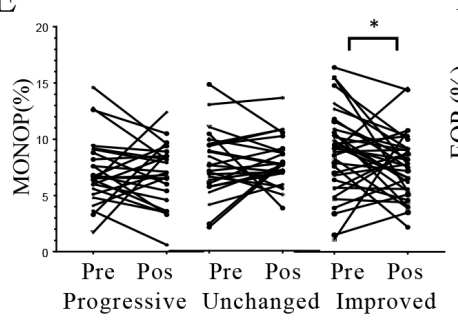

C

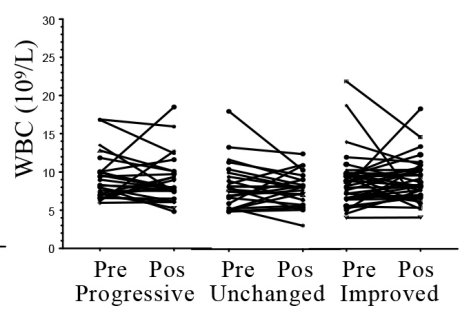

F

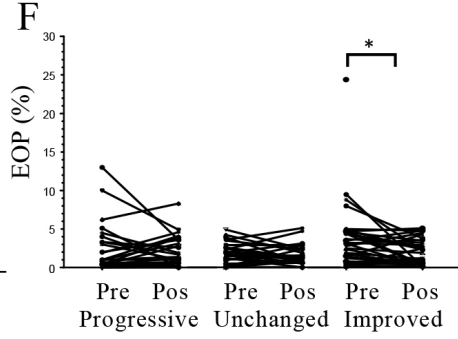

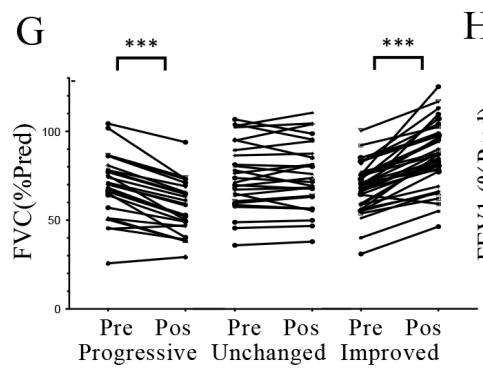
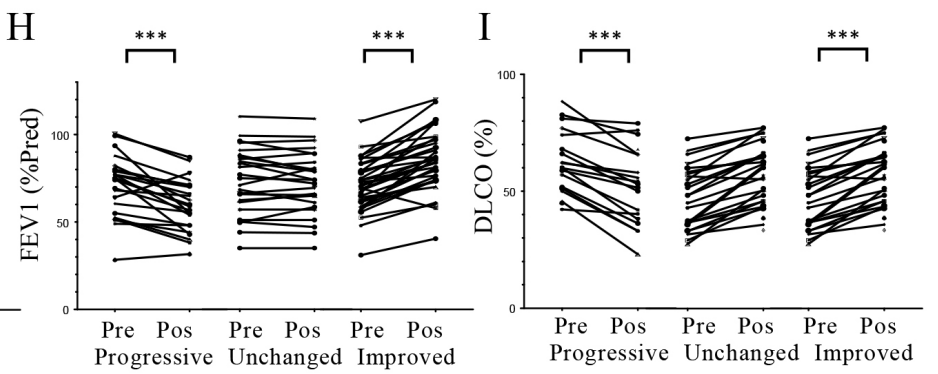

J
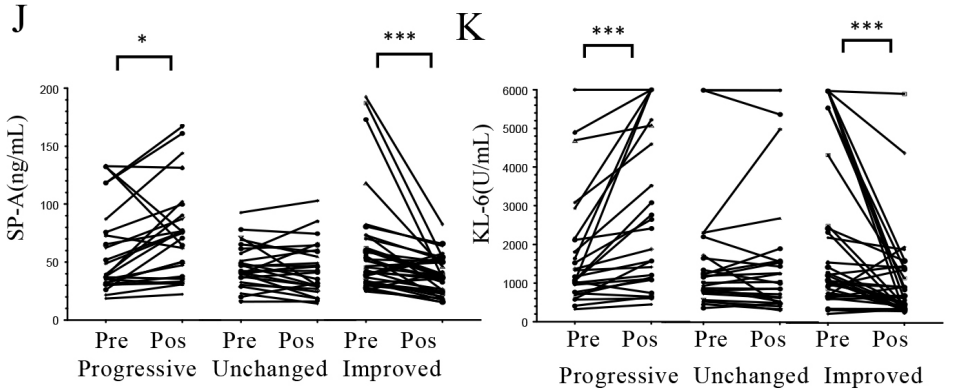

Figure 1: Comparison of the clinical and laboratory parameters between pretreatment and post-treatment. The patients were allocated to the progressive, unchanged, and improved groups according to the difference between pretreatment and post-treatment lung function. A: CRP levels; B: LDH levels; C: WBC count; D: NEUTP ratio; E: MONO ratio; F: EOP ratio; G: percent-predicted FVC (FVC \% Pred); H: percent-predicted FEV1 (FEV1 \% Pred); I: percent-predicted DLCO (DLCO \%); J: serum SP-A levels; K: serum KL-6 levels. Pre: pretreatment; Pos: post-treatment. $P$-values were calculated by Wilcoxon signed-rank U test. ${ }^{*} P<0.05,{ }^{*} P<0.005,{ }^{* *} P<0.001$. CRP: C-reactive protein; DLCO: diffusing capacity for carbon monoxide; EOP: eosinophil; FEV1: forced expiratory volume in 1 s; FVC: forced vital capacity; KL-6: Krebs von den Lungen-6; LDH: lactate dehydrogenase; MONO: monocyte; NEUTP: neutrophil; SP-A: surfactant protein-A; WBC: white blood cell.

$\mathrm{U} / \mathrm{mL}$ to 1875 (1110-5074) $\mathrm{U} / \mathrm{mL}(P<0.05)$. Serum levels of SP-A and KL-6 were above the cutoff level in $48.1 \%$ and $92.6 \%$ of patients, respectively, pretreatment; these ratios were $95.2 \%$ and $96.3 \%$, respectively, post-treatment (Table 1 and Figure 1J-K).

Conversely, in the improved group, there was a significant decrease in median serum SP-A levels from 45.9 (33.1-68.2) $\mathrm{ng} / \mathrm{mL}$ to $36.7(23.9-51.5) \mathrm{ng} / \mathrm{mL}(P<0.001)$ and in median serum KL-6 levels from $1169(729.3-3886.5) \mathrm{U} / \mathrm{mL}$ to 661 (394-1403) U/mL ( $P<0.05)$. Serum levels of SP-A and KL-6 were above the cutoff level in $52.7 \%$ and $91.7 \%$ of patients, respectively, before treatment; these ratios were $36.1 \%$ and $63.9 \%$, respectively, after treatment (Table 1 and Figure 1J-K).

In the unchanged group, there were no significant changes in median serum SP-A levels $(39.6[31.8-75.7] \mathrm{ng} / \mathrm{mL}$ vs. $38.6(27.3-57.9) \mathrm{ng} / \mathrm{mL} ; P>0.05)$ or in median serum KL-6 levels (1179 [781.5-2088.5] U/mL vs. 1023 


\begin{tabular}{|c|c|c|c|c|}
\hline Variables & Progression Group & Unchanged Group & Improvement Group & $P$-value \\
\hline Number (\%) & $27(29.7 \%)$ & $28(30.8 \%)$ & $36(39.5 \%)$ & - \\
\hline Follow-up (months) & $10.1(7.2-13.0)^{a}$ & $6.3(4.1-10.6)^{\mathrm{a}}$ & $8.1(4.4-12.4)^{a}$ & $0.057^{c}$ \\
\hline Age (years) & $55(48.0-65)^{\text {a }}$ & $50(41.3-66.8)^{a}$ & $55(44.3-61.0)^{a}$ & $0.876^{c}$ \\
\hline Male (\%) & $11(40.7 \%)$ & $15(53.6 \%)$ & $16(44.4 \%)$ & $0.612^{d}$ \\
\hline BMI $\left(\mathbf{k g} / \mathbf{m}^{2}\right)$ & $24.5(22.7-27.8)^{\text {a }}$ & $24.4(22.7-36.3)^{\text {a }}$ & $22.7(21.4-24.8)^{\mathrm{a}}$ & $0.161^{c}$ \\
\hline Smoker, n (\%) & $6(22.2 \%)$ & $8(28.6 \%)$ & $8(22.2 \%)$ & 0.808 \\
\hline WBC $\left(10^{9} / \mathrm{L}\right)$ & $8.2(6.9-10)^{\mathrm{a}}$ & $7.6(5.5-9.3)^{\mathrm{a}}$ & $8.1(6.6-9.7)^{\mathrm{a}}$ & $0.246^{c}$ \\
\hline NEUTP ratio (\%) & $62.5(51.2-69.5)^{\mathrm{a}}$ & $62.3(59.8-78.3)^{\text {a }}$ & $64.5(55.7-72.5)^{a}$ & $0.463^{c}$ \\
\hline MONO ratio (\%) & $6.7(5.6-9.1)^{\text {a }}$ & $7.6(6.1-9.5)^{\mathrm{a}}$ & $9.3(6.5-11.4)^{a}$ & $0.069^{c}$ \\
\hline EOP ratio $(\%)$ & $1(0.4-3.5)^{a}$ & $1.8(0.9-2.8)^{\text {a }}$ & $2.7(0.8-4.6)^{a}$ & $0.183^{c}$ \\
\hline BASOP ratio (\%) & $0.4(0.3-0.5)^{a}$ & $0.4(0.3-0.6)^{a}$ & $0.4(0.3-0.6)^{a}$ & $0.910^{c}$ \\
\hline $\operatorname{CRP}(\mathrm{ml} / \mathrm{dL})$ & $0.3(0.1-1.1)^{a}$ & $0.2(0.1-0.5)^{\text {a }}$ & $0.3(0.1-0.7)^{a}$ & $0.290^{c}$ \\
\hline LDH (U/L) & $229(180.6-258)^{\text {a }}$ & $202.9(179.1-252.3)^{a}$ & $202.3(165-223)^{\text {a }}$ & $0.187^{c}$ \\
\hline DLCO (\% predicted) & $59.4(51.2-69.5)^{\text {a }}$ & $49.8(40-62.5)^{a}$ & $56.0(43-69.3)^{a}$ & $0.072^{c}$ \\
\hline FVC (\% predicted) & $70.0(57.0-78.0)^{a}$ & $69.2(56.6-87.7)^{\mathrm{a}}$ & $68.8(58.5-75.4)^{\mathrm{a}}$ & $0.845^{c}$ \\
\hline FEV1 (\% predicted) & $75.3(57.8-79.6)^{\mathrm{a}}$ & $74.9(61.3-88.0)^{\text {a }}$ & $70.7(61.1-79.5)^{\mathrm{a}}$ & $0.586^{c}$ \\
\hline Pretreatment SP-A $(\mathrm{ng} / \mathrm{mL})$ & $41.7(33.5-55.9)^{\text {a }}$ & $39.6(31.8-75.7)^{\text {a }}$ & $45.9(33.1-68.2)^{\text {a }}$ & $0.645^{c}$ \\
\hline Post-treatment SP-A (ng/mL) & $75.1(37.6-90.6)^{\mathrm{a}}$ & $38.6(27.3-57.9)^{\text {a }}$ & $36.7(23.9-51.5)^{\text {a }}$ & $<0.001^{\mathrm{c}}$ \\
\hline Pretreatment KL-6 (U/mL) & $1070(738-2117)^{a}$ & $1179(781.5-2088.5)^{a}$ & $1169(729.3-3886.5)^{a}$ & $0.763^{c}$ \\
\hline Post-treatment KL-6 (U/mL) & $1875(1110-5074)^{a}$ & $1023(558-1820)^{\text {a }}$ & $661(394-1403)^{a}$ & $<0.001^{c}$ \\
\hline \multicolumn{5}{|l|}{ SP-A (> 43.8ng/mL) } \\
\hline Pretreatment & $13(48.1 \%)^{b}$ & $13(46.4 \%)^{b}$ & $19(52.7 \%)^{b}$ & $0.869^{d}$ \\
\hline Post-treatment & $25(95.2 \%)^{b}$ & $16(57.1 \%)^{b}$ & $13(36.1 \%)^{b}$ & $<0.001^{\mathrm{d}}$ \\
\hline \multicolumn{5}{|l|}{$\mathrm{KL}-6(>500 \mathrm{U} / \mathrm{mL})$} \\
\hline Pretreatment & $25(92.6 \%)$ & $26(92.8 \%)$ & $33(91.7 \%)$ & $1.000^{d}$ \\
\hline Post-treatment & $26(96.3 \%)$ & $22(78.6 \%)$ & $23(63.9 \%)$ & $0.009^{d}$ \\
\hline
\end{tabular}

a: media (IQR); b: Ratio (95\% IC); c: $P$-value was calculated by Kruskal-Wallis test; $\mathrm{d}$ : $P$-value was calculated by Fisher's exact test. BASOP: basophil count; BMI: body mass index; CRP: C-reactive protein; DLCO: diffusing capacity for carbon monoxide; EOP: eosinophil count; ILD: interstitial lung disease; FEV1: forced expiratory volume in 1 second; FVC, forced vital capacity; IC: Confidence Interval; IC: Confidence Interval; KL-6: Krebs von den Lungen-6; LDH: lactate dehydrogenase; NEUTP: neutrophil count; SP-A: surfactant protein-A; WBC: white blood cell count.

[558-1820] U/mL; $P>0.05)$. Serum levels of SP-A and KL-6 were above the cutoff level in $46.4 \%$ and $92.8 \%$ of patients, respectively, before treatment; these ratios were $57.1 \%$ and $78.6 \%$, respectively, after treatment (Table 1 and Figure $1 \mathrm{~J}-\mathrm{K})$.

\section{Correlations between changes in Delta KL-6 and Delta SP-A and changes in pulmonary function}

Figure 2 shows the results of the correlation analysis of the relationships between pretreatment and posttreatment changes in the serum biomarker levels (Delta SP-A and Delta KL-6) and predicted PFT parameters (Delta DLCO, Delta FVC, and Delta FEV1). Delta SP-A showed significant inverse correlations with Delta FVC (rs $=-0.231, P=0.001$ ) and Delta FEV1 (rs $=-0.339, P$
$=0.003)$. Similarly, Delta KL-6 showed significant inverse correlations with Delta FVC (rs $=-0.363, P<0.001)$ and Delta FEV1 (rs $=-0.550, P<0.001$ ). We also tested the correlation between Delta SP-A and Delta KL-6. This showed a significant positive correlation ( $\mathrm{rs}=0.523, \mathrm{P}$ $<0.001$; Figure 3). However, in the improved group, the number of patients with decreased SP-A and KL-6 levels was inconsistent.

\section{Cluster analysis based on the serum levels of SP-A and KL-6}

To explore the pathophysiological differences between KL-6 and SP-A, we classified 91 patients by cluster analysis and compared the pathophysiological characteristics between the clusters (Figure 4). The lung functions of 

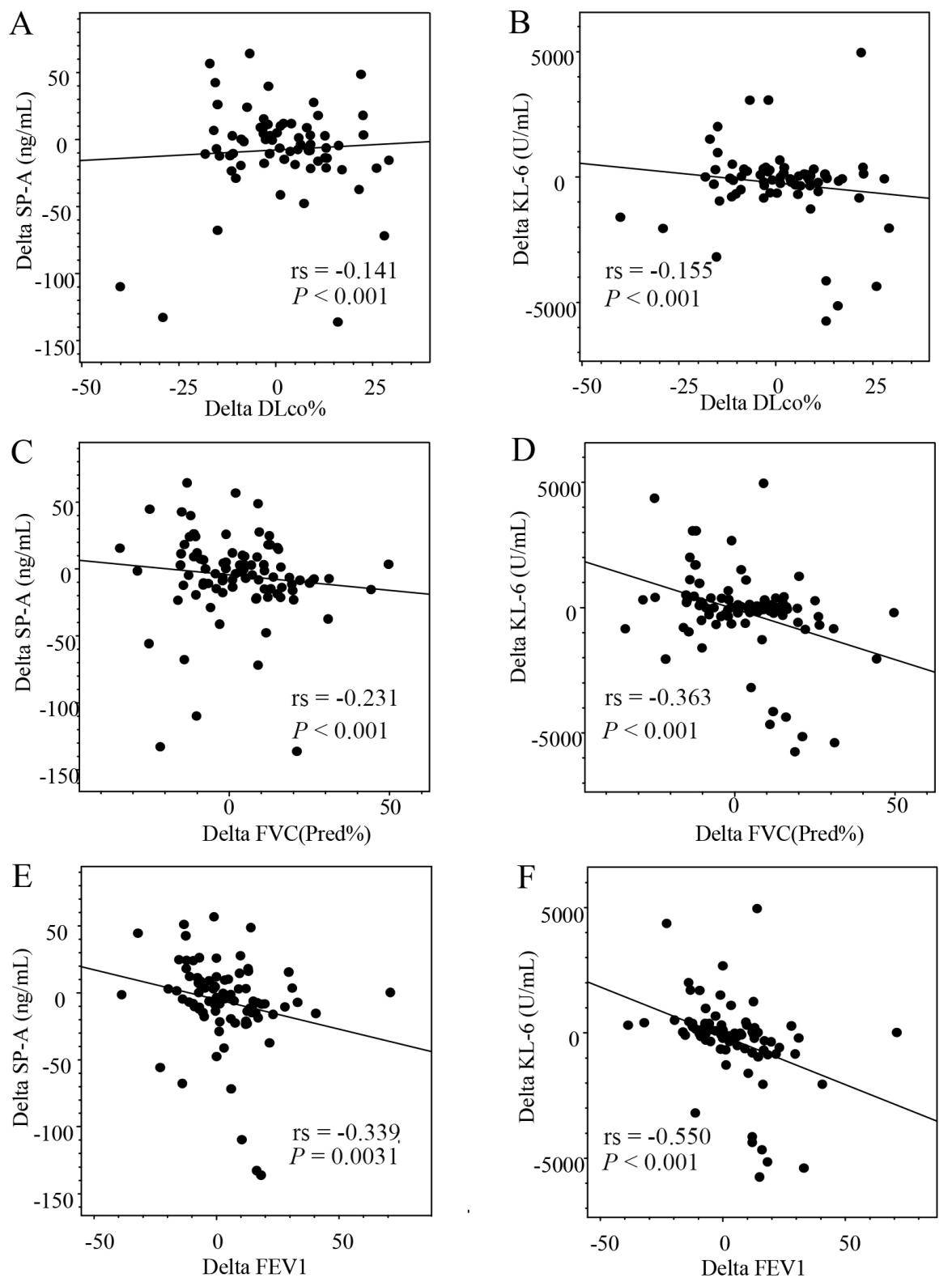

Figure 2: Correlations between Delta SP-A and Delta KL-6 and changes in pulmonary function test parameters. DLCO: diffusing capacity for carbon monoxide; FEV1: forced expiratory volume in 1 s; FVC: forced vital capacity; KL-6: Krebs von den Lungen-6; SP-A: surfactant protein-A.

all patients were tested for FVC. However, due to the difficulty in breathing, 19 patients were unable to cooperate with the DLCO test before or after treatment. Patients were classified into four groups (G1, G2, G3, and G4) based on the changes in KL-6 and SP-A levels. In G1 and G4, the levels of SP-A and KL-6 changed in the same direction: G1, SP-A and KL-6 increased; G4, SP-A and KL-6 decreased. In contrast, only the SP-A levels were reduced after treatment in G2, whereas only the SP-A levels were increased in G4 (Figure 5A, B). Pretreatment SP-A and KL-6 levels were significantly higher in G4 than in G3 (Figure 5C, D). Pretreatment EOP ratios did not differ significantly among the groups (Figure 5E). EOP ratios were significantly reduced in the G2 group (Figure 5F). Post-treatment DLCO (Pred\%), FVC (Pred\%), and FEV1 (Pred\%) were significantly improved in G4 (Figure $5 \mathrm{H}-\mathrm{J})$ and $\mathrm{FVC}(\operatorname{Pred} \%)$ was significantly improved in G1 (Figure 5J). In G1, 64.3\% of patients showed progression in disease activity and the remaining 35.7\% showed unchanged disease activity, and $86.4 \%$ of patients showed improved disease activity in G4 (Figure 5K). G2 showed significantly lower autoimmune disease frequency, whereas 95\% of G4 patients suffered autoimmune disease (Figure S2). The ratios of autoimmune diseases (Figure S2) were 


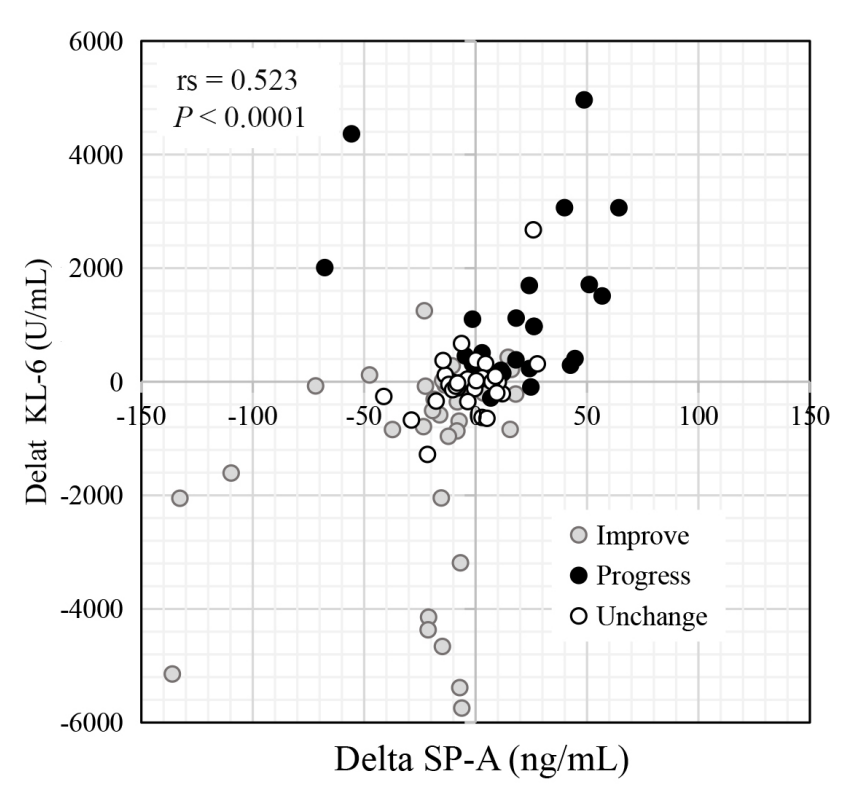

Figure 3: Correlation between serum Delta KL-6 and Delta SP-A values. The individual dots indicate patients in the unchanged (gray), progressive (black), and improved (open) groups. KL-6: Krebs von den Lungen-6; SP-A: surfactant protein-A.

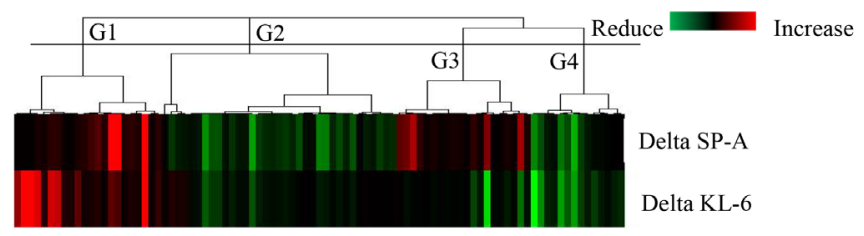

Figure 4: Unsupervised, hierarchical clustering analysis of Delta SP-A and Delta KL-6 levels. The cluster analysis was performed by centroid linkage based on correlation distance. Red represents increased biomarker levels and green represents decreased biomarker levels. The clustering analysis was used to divide the patients into four groups (G1, G2, G3, and G4). G1, G2, G3, and $\mathrm{G} 4$ are defined as statistically different groups by cluster analysis based on Delta KL-6 and SP-A values. G1: SP-A and KL-6 levels were increased; G2: only the SP-A levels were reduced; G3: only the SP-A levels were increased; G4: SP-A and KL-6 values were decreased; KL-6: Krebs von den Lungen-6; SP-A: surfactant protein-A.

significantly lower in G2 and higher in G4. The immune antifibrotic therapy ratio was not significantly different among the clusters (Figure 5L and Figure S3). Therefore, the correlation of the change in SP-A and KL-6 levels with the prognosis of the patients with ILD is mainly due to the response to steroids.

\section{DISCUSSION}

KL-6 is a mucin-like, high-molecular-weight glycoprotein that is strongly expressed in type II alveolar pneumocyte cells. It was discovered in 1985 by Kohno et al., ${ }^{[24]}$ and several studies have found it to be a serum marker of ILD. SP-A is a lung-specific protein that is produced by two types of epithelial cells in the peripheral airway: alveolar type II cells and Clara cells. ${ }^{[21]}$ Type II lung cells are alveolar wall cells that proliferate during lung injury repair. ${ }^{[7]}$ Elevated serum levels of SP-A and KL-6 reflect increased type II pneumocyte activity in the injured lung, with a resultant back leak into the blood. ${ }^{[6,9,18-20]}$ Serum KL-6 levels have been used in Japan as biomarkers in the diagnosis, severity assessment, and prediction of outcomes for patients with ILD. ${ }^{[3,4,18-20,25]}$ In patients with IPF, SP-A is a useful predictor of mortality and disease progression. ${ }^{[15]}$ It has been proposed that serum SP-A is a potential biomarker of antifibrotic drugs' therapeutic outcomes. ${ }^{[26]}$ Despite the fact that there are increasing reports about KL-6 and SP-A in the context of ILD, there has been little investigation into the difference between SP-A and KL-6 as biomarkers. This study investigated the characteristics of SP-A and KL-6 based on prognosis responses and evaluated their utility in disease monitoring.

We categorized a decline in FVC $\geq 10 \%$ or DLCO $\geq 15 \%$ as an indicator of disease progression and an increase in FCV $\geq 10 \%$ or DLCO $\geq 15 \%$ as disease improvement. Based on previous classifications, our criteria can be considered reasonable. ${ }^{[27,28]}$

In this study, EOP ratios showed a significant reduction after treatment in the improved group. EOPs are a type 2 (Th2) immune response component. As previously reported, Th2 immune responses are activated in some patients with ILD. ${ }^{[29,30]}$ Among the patients enrolled in this study, the EOP ratios were within normal ranges both before and after treatment. However, there is a possibility that Th2 inflammation of ILD-induced lowlevel eosinophilic inflammation was improved by the immunosuppressive treatment in the improved group of patients.

During pretreatment, $84 \%$ of patients showed higher serum KL-6 levels than the previously determined cutoff levels, whereas, for SP-A levels, only $49 \%$ of patients showed levels higher than the cut-off levels. The cut-off value of SP-A was defined by patients with IPF, and the sensitivity was reported to be $78.8 \% .{ }^{[29]}$ ROC analysis revealed that the best cut-off level of SP-A in the current study was $29 \mathrm{ng} / \mathrm{mL}$ (Figure S3). The patients were not limited to those with IPF and this may have affected the cut-off level of SP-A.

In this study, however, pretreatment serum levels of SP-A and KL-6 were not associated with disease prognosis. Pretreatment KL-6 levels were reported to be significantly different depending on the response to pirfenidone therapy for IPF, ${ }^{[31]}$ with only eight patients benefiting from it (Figure S4); the difference in treatment may have contributed to 

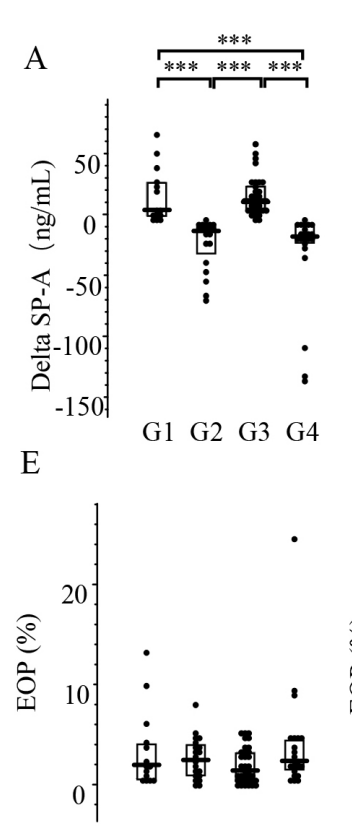

G1 G2 G3 G4

I

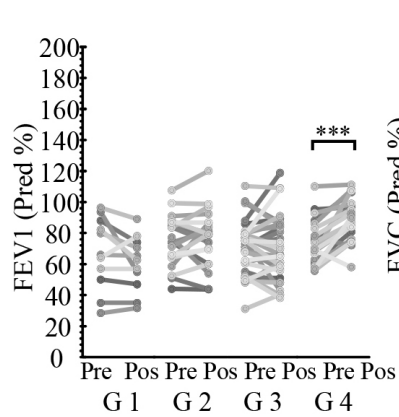

B
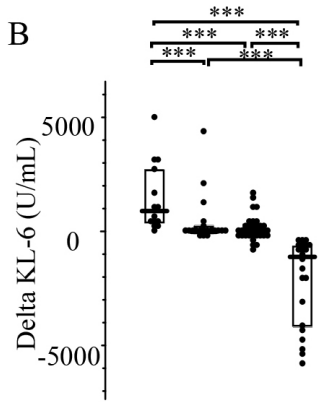

G1 G2 G3 G4

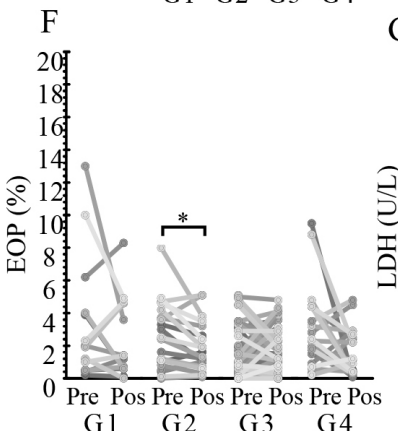

J
G1 G2 G3 G4
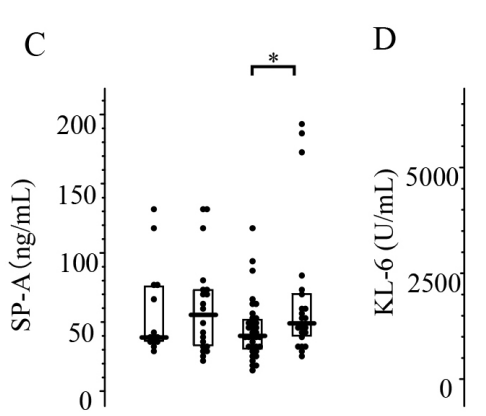

G

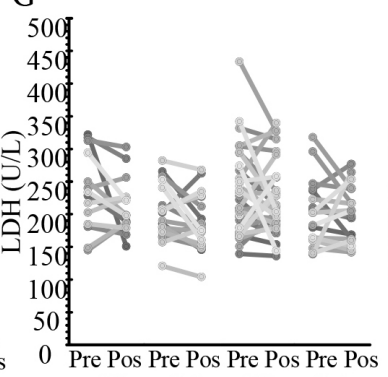

G1 G2 G3 G4

K

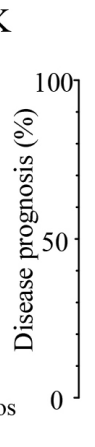

$P<0.001$

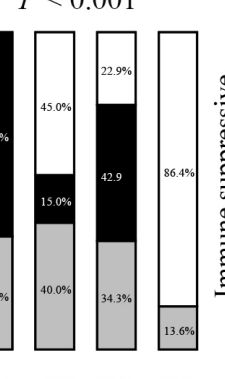

$\mathrm{H}$

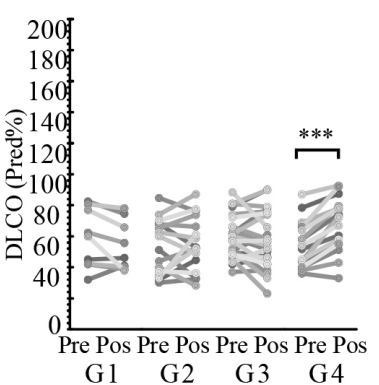

L

NS

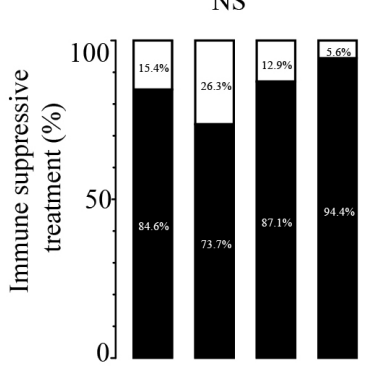

G1 G2 G3 G4

Figure 5: Comparison of respiratory function and prognosis among the three groups identified in the cluster analysis. A: Delta SP-A levels; B: Delta KL-6 levels; C: pretreatment serum SP-A levels; D: pretreatment serum KL-6 levels; E: pretreatment EOP; F: changes in blood EOP between pre- and post-treatment; G: changes in serum LDH levels between pre- and post-treatment; H: changes in percent-predicted DLCO pre- and post-treatment; I: changes in percent-predicted FVC pre- and post-treatment; J: changes in percent-predicted FEV1 pre- and post-treatment; K: differences in disease prognosis between the three groups (black: progressive group; gray: unchanged group; and white: improved group); L: differences in immunosuppressive treatment between the three groups (black: with immune suppressive treatment; white: without immune suppressive treatment). A-E, the results are presented as individual data points with medians (bars) and interquartile ranges (boxes). $P$-values were calculated by Steel-Dwass test (A-E), Wilcoxon signed-rank test (F-J), and Fisher's exact test (K, L). DLCO: diffusing capacity for carbon monoxide; EOP: eosinophil ratio; FEV1: forced expiratory volume in 1 s; FVC: forced vital capacity; LDH: lactate dehydrogenase; KL-6: Krebs von den Lungen-6; SP-A: surfactant protein-A.

this discrepancy. In contrast, our study showed that the changes in serum levels of SP-A and KL-6 correlated significantly with changes in respiratory function, which reflects disease activity. So far, both SP-A and KL-6 have been identified as markers that reflect the therapeutic effect. ${ }^{[6,10,11,26,28,31-36]}$ However, there is a scarcity of data describing the differences in therapeutic response of each marker for individual patients. As mentioned above, these differences may reflect the endotype of the patient and are important for understanding the pathophysiology of each patient. This evidence emphasizes the importance of biomarkers in monitoring the activity of ILDs, considering how difficult it is to conduct respiratory function tests in patients with ILD.

Although there was a significant correlation between Delta SP-A and Delta KL-6, the correlation coefficient was not insufficient, implying that each marker represents a different pathophysiology. Ishii et al. ${ }^{[4]}$ reported that serum SP-A levels were higher in usual interstitial pneumonitis and lower in nonspecific interstitial pneumonia, whereas KL-6 levels were higher in both. Yoshikawa et al. ${ }^{[26]}$ also indicated that changes in serum SP-A levels reflected the outcomes of antifibrotic drug 
therapy more strongly than KL-6 levels did. SP-A is produced primarily in Clara cells and type II alveolar cells, whereas KL-6 is produced only in type II alveolar cells. ${ }^{[10,14,24]} \mathrm{SP}-\mathrm{A}$ is a C-type lectin with a molecular weight of $26-38 \mathrm{kDa}$, whereas KL-6 is a mucin-like glycoprotein with a large molecular weight of $200 \mathrm{kDa} \cdot{ }^{[14,37]}$ Biological and biochemical differences between SP-A and KL-6 are expected to be associated with different pathological changes in ILD.

To elucidate the pathophysiological differences between SP-A and KL-6, the patients were divided into four groups (G1, G2, G3, and G4) using cluster analysis, according to the values of Delta SP-A and Delta KL-6, and the pathophysiological characteristics were compared between the clusters. SP-A and KL-6 levels were both elevated in the G1 group with all the patients enrolled classified as either progressive or unchanged. Despite the higher immunosuppressive treatment ratio in this group, the respiratory function decreased, suggesting that noninflammatory mechanisms, such as fibrosis, may contribute to the pathophysiology.

The G4 group had lower levels of SP-A and KL-6, and the patients had better respiratory function. Approximately $94.4 \%$ of the patients were treated with immunosuppressive treatment. In the G2 group, only the SP-A levels decreased, corresponding to an improvement in respiratory function. Interestingly, EOP ratios of G2 were significantly reduced after treatment (Figure 5F). These patients might be sensitive to immunosuppressive treatment because of their dependence on Th2 inflammatory pathophysiology. Previous research has suggested that serum levels of $\mathrm{LDH}$ can be used to monitor disease activity and progression and are considered to reflect pulmonary cell damage. ${ }^{[38]}$ SP-A levels were reported to be elevated in the acute phase of acute respiratory distress syndrome caused by coronavirus disease 2019 infection, compared to what is commonly seen during a relatively early stage of pneumonia. ${ }^{[39]}$ Serum SP-A levels have been linked to acute exacerbations of ILD, whereas KL-6 levels are elevated in drug-induced pneumonia or CTD-ILD. ${ }^{[13]}$ In addition, SP-A expression was equally reported to correlate negatively with the fibrosis score. ${ }^{[40]}$ Takahashi et al. ${ }^{[10]}$ reported that SP-A levels, rather than the indicators of the end stage of fibrotic changes, such as honeycombing, were associated with reversible ground-glass opacity levels. They also reported that SP-A levels were significantly lower in parenchymal collapse opacity-dominant type patients than in ground-glass opacity-dominant type patients. ${ }^{[10]}$ Studies have shown that serum SP-A levels rise faster than KL-6 in the progressive group. ${ }^{[2]}$ This suggests that G2 patients were not at the end stage of the disease and that their respiratory function improved with immunosuppressive treatment. Similarly, the patients of G3 might be in an early stage of disease progression. Therefore, SP-A and KL-6 are suitable to monitor changes in different pathological types of ILD. We consider that the relationship between changes in SP-A and KL-6 levels and the treatment response of this study is primarily a response to the degree of lung tissue damage and the stage of disease progression. Moreover, the lower molecular weight of SP-A compared to KL-6 may provide an advantage in detecting it in blood serum at an earlier stage of ILD. In summary, while both SP-A and KL-6 are pathological markers that reflect the disease course in patients with ILD, only SP-A levels responded to disease improvement in some patients. It is, therefore, important to simultaneously measure both SP-A and KL-6 levels for the pathophysiological monitoring of patients with ILD.

This study had several limitations. First, we included patients with definite typical interstitial pneumonitis patterns on HRCT but without surgical lung biopsies, making accurate classification of ILD subtype difficult. Second, because this was a retrospective study, the observation period varied between patients. Finally, due to a lack of surgical lung biopsies, we were unable to compare the results of serum biomarkers to histopathologic patterns. Future prospective studies are needed to clarify the ILD type-related behaviors and prognostic factors found in these serum biomarkers.

\section{CONCLUSIONS}

Our results demonstrated that serum levels of SP-A and KL-6 are significantly lower in those patients with ILD who showed disease improvement and significantly higher in those with ILD who showed disease progression. Because the responses of both markers differed based on their pathophysiological or biological characteristics, measuring both markers is crucial for understanding a patient's pathophysiological condition.

\section{Ethics approval and consent to participate}

Ethical Statement: This study was approved by the Ethics Committee of the First Affiliated Hospital of Guangzhou Medical University (approval number: GYFYY-2016-73).

The use of human serum samples was in accordance with relevant legislation in China and the wishes of donors, their legal guardians, or the next of kin, where applicable, who had offered written informed consent to use the serum samples for future unspecified research purposes.

\section{Consent for publication}


Not applicable.

\section{Competing interests}

The authors declare that they have no competing interests.

\section{Authors' contributions}

(I) Conception and design: B Sun; (II) administrative support: B Sun and P Zheng; (III) provision of study materials or patients: P Zheng, X Zheng, J Wang; (IV) collection and assembly of data: X Zheng; (V) data analysis and interpretation: $\mathrm{H}$ Takehiro, X Zheng, P Zheng, Z Huang; (VI) manuscript writing: all authors; and (VII) final approval of the manuscript: all authors.

\section{Acknowledgment}

Funding from Sysmex Corp. is gratefully acknowledged.

\section{Source of Funding}

This study was funded by the National Natural Science Foundation of China (Project No.: 81871736), Guangdong Science and Technology Fund (Project No.: 2020B1111300001), Guangzhou Institute of Respiratory Health Open Project (funds provided by China Evergrande Group) (Project No.:2020GIRHHMS04), and Science and Technology Project of Guangzhou (Project No.: 20181A011061). Cultivation Project of the First Affiliated Hospital of Guangzhou Medical University (Project No.: ZH201915, ZH201818) and Non-profit Central Research Institute Fund of Chinese Academy of Medical Sciences (Project No.: 2018PT31048).

\section{REFERENCES}

1. Huapaya JA, Wilfong EM, Harden CT, Brower RG, Danoff SK. Risk factors for mortality and mortality rates in interstitial lung disease patients in the intensive care unit. European Respiratory Review 2018; 27:180061.

2. Meyer KC. Diagnosis and management of interstitial lung disease. Transl Respir Med 2014; $2: 4$.

3. Ishikawa N, Hattori N, Yokoyama A, Kohno N. Utility of KL-6/MUC1 in the clinical management of interstitial lung diseases. Respir Investig 2012; 50: 3-13.

4. Ishii H, Mukae H, Kadota J, Kaida H, Nagata T, Abe K, et al. High serum concentrations of surfactant protein A in usual interstitial pneumonia compared with non-specific interstitial pneumonia. Thorax 2003; 58: $52-7$.

5. Hamai K, Iwamoto H, Ishikawa N, Horimasu Y, Masuda T, Miyamoto S, et al. Comparative Study of Circulating MMP-7, CCL18, KL-6, SP-A, and SP-D as Disease Markers of Idiopathic Pulmonary Fibrosis. Disease Markers 2016; 2016: 4759040.

6. Ohnishi H, Yokoyama A, Kondo K, Hamada H, Abe M, Nishimura K, et al. Comparative study of KL-6, surfactant protein-A, surfactant protein-D, and monocyte chemoattractant protein-1 as serum markers for interstitial lung diseases. Am J Respir Crit Care Med 2002; 165: 378-81.
7. Chiba H, Otsuka M, Takahashi H. Significance of molecular biomarkers in idiopathic pulmonary fibrosis: A mini review. Respir Investig 2018; 56: 384-91.

8. Samukawa T, Hamada T, Uto H, Yanagi M, Tsukuya G, Nosaki T, et al. The elevation of serum napsin A in idiopathic pulmonary fibrosis, compared with KL-6, surfactant protein-A and surfactant protein-D. BMC Pulm Med 2012; 12: 55.

9. Cho EJ, Park KJ, Ko DH, Koo HJ, Lee SM, Song JW, et al. Analytical and Clinical Performance of the Nanopia Krebs von den Lungen 6 Assay in Korean Patients With Interstitial Lung Diseases. Ann Lab Med 2019; 39: 245-51.

10. Takahashi H, Fujishima T, Koba H, Murakami S, Kurokawa K, Shibuya $\mathrm{Y}$, et al. Serum surfactant proteins $\mathrm{A}$ and $\mathrm{D}$ as prognostic factors in idiopathic pulmonary fibrosis and their relationship to disease extent. Am J Respir Crit Care Med 2000; 162: 1109-14.

11. Nakamura M, Okamoto M, Fujimoto K, Ebata T, Tominaga M, Nouno $\mathrm{T}$, et al. A retrospective study of the tolerability of nintedanib for severe idiopathic pulmonary fibrosis in the real world. Ann Transl Med 2019; $7: 262$.

12. Kakugawa T, Yokota $S$, Ishimatsu Y, Hayashi T, Nakashima S, Hara S, et al. Serum heat shock protein 47 levels in patients with drug-induced lung disease. Respir Res 2013; 14: 133.

13. Kitajima H, Takahashi H, Harada K, Kanai A, Inomata S, Taniguchi H, et al. Gefitinib-induced interstitial lung disease showing improvement after cessation: disassociation of serum markers. Respirology 2006; 11 : 217-20.

14. Kuroki Y, Takahashi H, Chiba H, Akino T. Surfactant proteins A and D: disease markers. Biochim Biophys Acta 1998; 1408: 334-45.

15. Song JW, Do KH, Jang SJ, Colby TV, Han S, Kim DS. Blood biomarkers MMP-7 and SP-A: predictors of outcome in idiopathic pulmonary fibrosis. Chest 2013; 143: 1422-9.

16. Xue M, Guo Z, Cai C, Sun B, Wang H. Evaluation of the Diagnostic Efficacies of Serological Markers KL-6, SP-A, SP-D, CCL2, and CXCL13 in Idiopathic Interstitial Pneumonia. Respiration 2019; 98: 534-45.

17. Zhang Y, Kaminski N. Biomarkers in idiopathic pulmonary fibrosis. Curr Opin Pulm Med 2012; 18: 441-6.

18. Bonella F, Volpe A, Caramaschi P, Nava C, Ferrari P, Schenk K, et al. Surfactant protein D and KL-6 serum levels in systemic sclerosis: correlation with lung and systemic involvement. Sarcoidosis Vasc Diffuse Lung Dis 2011; 28: 27-33.

19. Hant FN, Ludwicka-Bradley A, Wang HJ, Li N, Elashoff R, Tashkin DP, et al. Surfactant Protein D and KL-6 as Serum Biomarkers of Interstitial Lung Disease in Patients with Scleroderma. Journal of Rheumatology 2009; 36: 773-80.

20. Benyamine A, Heim X, Resseguier N, Bertin D, Gomez C, Ebbo M, et al. Elevated serum Krebs von den Lungen-6 in systemic sclerosis: a marker of lung fibrosis and severity of the disease. Rheumatology International 2018; 38: 813-9.

21. Wang K, Ju Q, Cao J, Tang WZ, Zhang J. Impact of serum SP-A and SP-D levels on comparison and prognosis of idiopathic pulmonary fibrosis: A systematic review and meta-analysis. Medicine 2017; 96:e7083.

22. Zheng PY, Liu XQ, Huang HM, Guo ZJ, Wu G, Hu HS, et al. Diagnostic value of KL-6 in idiopathic interstitial pneumonia. Journal of Thoracic Disease 2018; 10:4724-32.

23. Fischer A, Antoniou KM, Brown KK, Cadranel J, Corte TJ, du Bois RM, et al. An official European Respiratory Society/American Thoracic Society research statement: interstitial pneumonia with autoimmune features. European Respiratory Journal 2015; 46: 976-87.

24. Kohno N, Kyoizumi S, Awaya Y, Fukuhara H, Yamakido M, Akiyama M. New serum indicator of interstitial pneumonitis activity. Sialylated carbohydrate antigen KL-6. Chest 1989; 96: 68-73.

25. Arai S, Kurasawa K, Maezawa R, Owada T, Okada H, Fukuda T. Marked increase in serum KL-6 and surfactant protein D levels during the first 4 weeks after treatment predicts poor prognosis in patients with active 
interstitial pneumonia associated with polymyositis/dermatomyositis. Mod Rheumatol 2013; 23: 872-83.

26. Yoshikawa T, Otsuka M, Chiba H, Ikeda K, Mori Y, Umeda Y, et al. Surfactant protein A as a biomarker of outcomes of anti-fibrotic drug therapy in patients with idiopathic pulmonary fibrosis. BMC Pulm Med 2020; 20: 27.

27. Cottin V, Wollin L, Fischer A, Quaresma M, Stowasser S, Harari S. Fibrosing interstitial lung diseases: knowns and unknowns. European Respiratory Review 2019; 28:180100.

28. Lee YS, Kim HC, Lee BY, Lee CK, Kim MY, Jang SJ, et al. The Value of Biomarkers as Predictors of Outcome in Patients with Rheumatoid Arthritis-Associated Usual Interstitial Pneumonia. Sarcoidosis Vasculitis and Diffuse Lung Diseases 2016; 33: 216-23.

29. Kishi M, Miyazaki Y, Jinta T, Furusawa H, Ohtani Y, Inase N, et al. Pathogenesis of cBFL in common with IPF? Correlation of IP-10/TARC ratio with histological patterns. Thorax 2008; 63: 810-6.

30. Nukui Y, Yamana T, Masuo M, Tateishi T, Kishino M, Tateishi U, et al. Serum CXCL9 and CCL17 as biomarkers of declining pulmonary function in chronic bird-related hypersensitivity pneumonitis. Plos One 2019; 14 .

31. Ikeda K, Shiratori M, Chiba H, Nishikiori H, Yokoo K, Saito A, et al. Serum surfactant protein D predicts the outcome of patients with idiopathic pulmonary fibrosis treated with pirfenidone. Respiratory Medicine 2017; 131: 184-91.

32. Hu Y, Wang LS, Jin YP, Du SS, Du YK, He X, et al. Serum Krebs von den Lungen-6 level as a diagnostic biomarker for interstitial lung disease in Chinese patients. Clinical Respiratory Journal 2017; 11: 337-45.

33. Hu CJ, Wu CY, Yang EH, Huang H, Xu D, Hou Y, et al. Serum KL-6 is associated with the severity of interstitial lung disease in Chinese patients with polymyositis and dermatomyositis. Clinical Rheumatology 2019; 38: 2181-7.

34. Cao XY, Hu SS, Xu D, Li MT, Wang Q, Hou Y, et al. Serum levels of Krebs von den Lungen- 6 as a promising marker for predicting occurrence and deterioration of systemic sclerosis-associated interstitial lung disease from a Chinese cohort. International Journal of Rheumatic Diseases 2019; 22: 108-15.

35. Lee JS, Lee EY, Ha YJ, Kang EH, Lee YJ, Song YW. Serum KL-6 levels reflect the severity of interstitial lung disease associated with connective tissue disease. Arthritis Research \& Therapy 2019; 21:58.

36. Yamakawa H, Hagiwara E, Kitamura H, Yamanaka Y, Ikeda S, Sekine A, et al. Serum KL-6 and surfactant protein-D as monitoring and predictive markers of interstitial lung disease in patients with systemic sclerosis and mixed connective tissue disease. Journal of Thoracic Disease 2017; 9: $362-71$

37. Brayman M, Thathiah A, Carson DD. MUC1: a multifunctional cell surface component of reproductive tissue epithelia. Reprod Biol Endocrinol 2004; 2: 4.

38. Tzouvelekis A, Kouliatsis G, Anevlavis S, Bouros D. Serum biomarkers in interstitial lung diseases. Respir Res 2005; 6: 78.

39. Saito A, Kuronuma K, Moniwa K, Kodama K, Takahashi S, Takahashi $\mathrm{H}$, et al. Serum surfactant protein A and D may be novel biomarkers of COVID-19 pneumonia severity. Research Square 2020.

40. Nagata N, Kitasato Y, Wakamatsu K, Kawabata M, Fukushima K, Kajiki $\mathrm{A}$, et al. Prognostic value of immunohistochemical surfactant protein A expression in regenerative/hyperplastic alveolar epithelial cells in idiopathic interstitial pneumonias. Diagnostic Pathology 2011; 6:25.

How to cite this article: Zheng $\mathrm{P}$, Zheng $\mathrm{X}$, Takehiro H, Cheng ZJ, Wang J, Xue M, et al. The prognostic value of Krebs von den Lungen-6 and surfactant protein-A levels in the patients with interstitial lung disease. J Transl Intern Med 2021; 9: 212-22. 